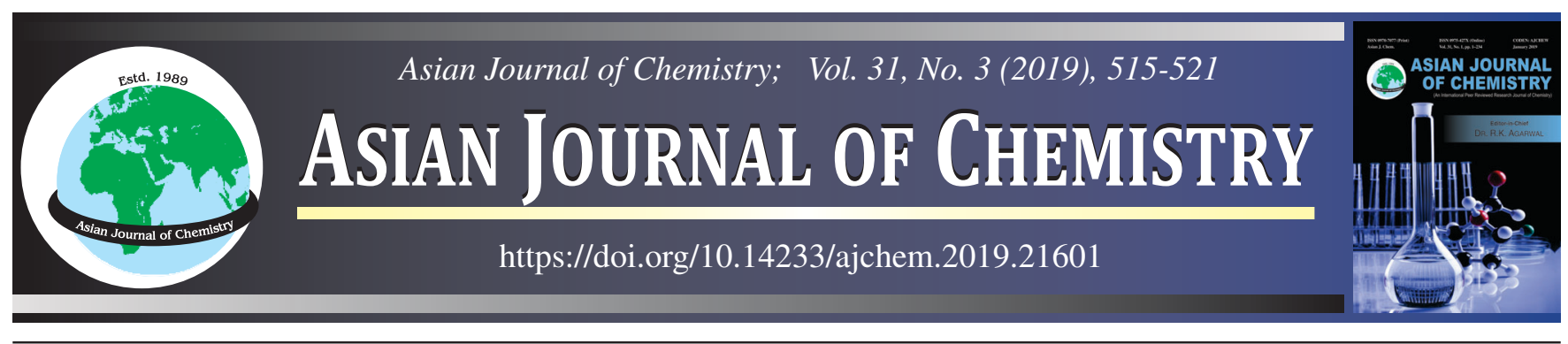

\title{
Assessment of Ground Water Contamination by Inorganic Impurities in Ferozepur District of Punjab State, India
}

\author{
GurJeet Kaur ${ }^{1}$, Sangeeta Sharma ${ }^{1, *}$ and Umesh Kumar Garg ${ }^{2}$
}

${ }^{1}$ Department of Applied Sciences and Humanities, Shaheed Bhagat Singh State Technical Campus, Ferozepur-152001, India

${ }^{2}$ Guru Teg Bahudar Khalsa College of Information Technology, Chhapianwali, Malout-152107, India

*Corresponding author: E-mail: sangeetassharma70@gmail.com

Received: 23 July 2018;

Accepted: 22 August 2018;

Published online: 31 January 2019;

AJC-19237

Malwa region of Punjab state, India has become the center of water borne diseases due to excessive use of pesticides, chemical fertilizers,
heavy metals, industrial toxins that cause toxicity in water. The main contamination in ground water is by physico-chemical parameters
and heavy metals $i . e$. $\mathrm{pH}$, total dissolved solids, total alkalinity, total hardness, calcium, chlorides, fluorides, arsenic and lead. The
contamination of ground water with heavy metals causes health hazards to humans and animals. Due to lack of adequate facilities and
resources for the management and handling of waste, the ground water contamination has been increased. In the present study, assessment
of ground water quality was carried out in the villages of Ferozepur district of Punjab state, India. With main emphasis on analyzing the
groundwater parameters of Ferozepur district which are responsible for health hazard to humans and animals. Various groundwater
samples were collected randomly from the villages of Ferozepur district and analyzed for pH, total dissolved solids, total alkalinity, total
hardness, calcium, chlorides, fluorides, heavy metals (arsenic and lead) using standard procedures. The concentrations of calcium, chlorides,
fluorides and pH were within the permissible limits, whereas, alkalinity and total hardness were observed beyond permissible limits in
most of the water samples. Even among majority of the samples taken, the concentration of arsenic and lead was found within the permissible
limits. Results showed that the ground water samples collected from depth ranging from 100 to $360 \mathrm{ft,} \mathrm{recorded} \mathrm{values} \mathrm{within} \mathrm{permissible}$
limits for drinking purpose as prescribed by WHO. Further, ANOVA has been applied on analysis results to study the effect of pH on
fluoride and chloride, depth on fluoride and chloride and depth on arsenic and lead. Also, to adjudge the overall quality of water in
Ferozepur district, the water quality index (WQI) has been calculated on the basis of large number of physico-chemical characteristics of
water. The water quality index of ground water in Ferozepur district has been calculated to be 107 . The value is close to 100 so the quality
of ground water in Ferozepur district can be categorized under 'Good Quality' water.

Keywords: Groundwater, Water quality index, Contamination, Inorganic pollutants.

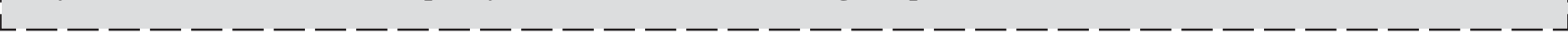

\section{INTRODUCTION}

Water is a primary need for humans and crucial natural resource [1]. There is 70-80\% water in human body. The general survey reveals that 36.1 crore $\mathrm{km}^{2}$ surface area is covered by sea out of total surface area of earth i.e. 51 crore $\mathrm{km}^{2}$. Rivers, lakes and groundwater are the sources of fresh water. The availability of fresh water is getting less day by day in the world [2]. In India, $89 \%$ of groundwater is used for irrigation purpose which is the major source of consumption of groundwater, whereas $9 \%$ is used for domestic purpose and 2 $\%$ for industrial purpose [3].
Hydrological monitoring stations are the source of determination of quantity of water by maintaining record of water level, its discharge and velocity and water quality is determined by collecting water samples from points, fixed at these monitoring stations and analysis of various parameters is done. The analysis results revealed the quality of water, on the basis of which spatial and temporal trends of surface water and groundwater are obtained [4].

Groundwater is stored underground in aquifers and is available below the surface in rocks and soil. About $97 \%$ of freshwater is mainly groundwater and is a vital source of drinking water all-over the world. In areas, where surface water supply

This is an open access journal, and articles are distributed under the terms of the Creative Commons Attribution-NonCommercial-ShareAlike 4.0 (CC BY-NC-SA 4.0) International License which allows readers to freely read, download, copy, distribute, print, search, or link to the full texts of its articles and to use them for any other lawful non-commercial purpose as long as the original source is duly acknowledged. 
is less and polluted, groundwater is the only source of drinking water [5]. Groundwater at depth is usually safe from microorganisms and chemicals where direct contamination is not possible; whereas at shallow, contamination of groundwater occur by mixing of sewerage, industrial waste and agriculture waste, pesticides, etc. [6].

In Punjab state, groundwater is reducing day by day because of its misuse and mishandling and its contamination by geogenic and anthropogenic sources [7]. Contamination of groundwater also occurs due to leaching of fertilizers and manures from agriculture fields. The untreated effluents discharged by industries into sewers/drains may lead to contamination of groundwater as untreated effluents have limits more than permissible limits prescribed by the Government for discharge onto land for plantation/irrigation. The presence of heavy metals in drinking water also causes detrimental impacts on human health if present in higher side than a certain concentration [8].

For maintaining the health of ecosystems, the quality of water is a necessary parameter. The physical, chemical, biological properties and availability of water influence the health of ecosystems. Some ecosystems are sensitive and can be degraded by small changes in the physical and chemical properties of the water [4]. Punjab's ecosystem is drastically affected from contamination of groundwater by various sources like geogenic, agricultural, industrial and urban activities which leads to reduction of groundwater resources and contaminates soil, plants, animals and humans. Keeping in view, all the above aspects, the present study involves the assessment of groundwater quality with respect to inorganic pollutants of Ferozepur district of Punjab state, India.

\section{EXPERIMENTAL}

Study area: Firozpur district of Punjab state, India occupies an area of about 5,305 $\mathrm{km}^{2}$ in southwestern Punjab (India). Total population of the district is about 10 lakhs, out of which, 5 lakhs are male and 5 lakhs are female. About $95 \%$ rural population depends upon groundwater for execution of daily routine activities like drinking, domestic and agricultural purposes. Global positioning system is used for determining the geographical coordinates of the study area. The coordinates of total study area lie between latitudes of $30^{\circ} 49.218^{\prime}$ and $30^{\circ} 57.003^{\prime}$ north and longitudes of $74^{\circ} 23.078^{\prime}$ and $74^{\circ} 55.638^{\prime}$ east (Table-1).

Topography: The state of Punjab has been classified into five agro-climatic zones on the basis of homogeneity, rainfall pattern and distribution, temperature, soil texture and cropping pattern (Fig. 1). Out of the five agro-climatic zones, Ferozepur district fall under western plain zone, which is characterized by arid (dry) and hot zone. Average annual temperature ranges from $25^{\circ} \mathrm{C}$ to $26^{\circ} \mathrm{C}$ and mean annual rainfall varies from 200 $\mathrm{mm}$ to $400 \mathrm{~mm}$. Depth to water level in the area ranges from $1.5 \mathrm{~m}$ to $31 \mathrm{~m}$ below ground level (mbgl). Water table is at shallow depth at several areas of Ferozepur district where it ranges from $1.5 \mathrm{mbgl}$ to $7.5 \mathrm{mbgl}$ causing water logging at many places. The groundwater is mainly abstracted through hand pumps (up to $25 \mathrm{~m}$ ) and shallow and medium depth tube wells (upto $175 \mathrm{~m}$ ) [9].

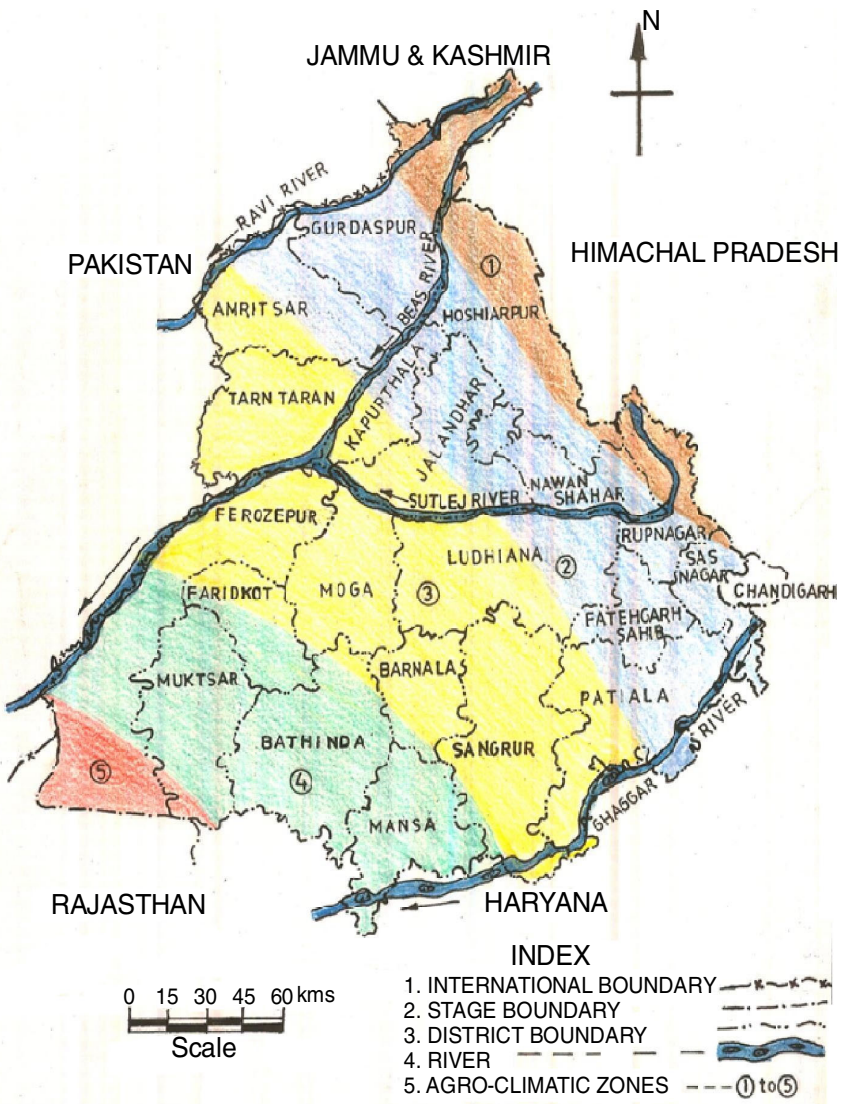

Fig. 1. Showing five agro climatic zones of Firozpur district of Punjab

Sampling: The primary data regarding extent of various inorganic impurities in the groundwater was collected in the form of questionnaire drafted in the local language for the ease of people. It consisted of questions related to the water source (type, age and bore depth as well as queries about the use of water for drinking and domestic purposes or agricultural purposes). Number of persons surveyed for each source depended on the source of water as well as the medical history of various inorganic impurities in the particular area. By visiting the selected areas of the district, 66 drinking water samples were collected from underground sources where hand pumps, tube wells and submersible pumps were installed. All the samples were stored in identical PET bottles (1 L capacity), marked numerically, and then various parameters were tested namely, $\mathrm{pH}$, TDS, alkalinity, total hardness, and for the content of fluoride, chloride, arsenic, and lead. The analysis results were compared with acceptable limit of WHO for drinking water.

Analysis: Physico-chemical parameters such as pH, TDS, alkalinity, total hardness, fluoride, and chloride concentration were analyzed by using standard methods. $\mathrm{pH}$ of solution is determined by using portable handy $\mathrm{pH}$ meter, TDS by using TDS meter and fluoride by using field test kits of Merck. The collected samples were also tested for fluoride concentration in water in the laboratory and results were cross-checked by colorimetric determination using SPADNS method, TDS by gravimetric method, total hardness by EDTA titrimetric method, chloride by argentometric titrimetric method, arsenic by atomic absorption spectrometry using hydride vapour generation technique and lead by AAS using graphite furnace assembly (Shimadzu model AA-7000). 
PHYSICO-CHEMICAL CHARACTERISTICS OF GROUNDWATER/ DRINKING WATER

\begin{tabular}{|c|c|c|c|c|c|c|c|c|c|c|c|c|c|c|c|}
\hline \multirow{2}{*}{$\begin{array}{l}\text { S. } \\
\text { No. }\end{array}$} & \multirow{2}{*}{$\stackrel{\substack{\infty \\
=}}{=}$} & \multirow{2}{*}{ 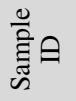 } & \multirow{2}{*}{$\begin{array}{l}\text { Depth } \\
\text { (feet) }\end{array}$} & \multirow[b]{2}{*}{ Source (age) } & \multirow[b]{2}{*}{ Latitude } & \multirow[b]{2}{*}{ Longitude } & & & & & illg, & & & & \\
\hline & & & & & & & $\mathrm{pH}$ & TDS & $\mathrm{F}^{-}$ & Alkalinity & $\mathrm{TH}$ & $\mathrm{Ca}^{2+}$ & $\mathrm{Cl}^{-}$ & $\mathrm{As}^{3+}$ & $\mathrm{Pb}^{2+}$ \\
\hline 1 & V1 & V1A & 45 & HP 2000 & $\mathrm{~N} 30^{\circ} 49.649^{\prime}$ & $\mathrm{E}^{\prime} 4^{\circ} 45.453^{\prime}$ & 7.8 & 950 & 1.10 & 436 & 264 & 30 & 116 & 0.010 & 0.010 \\
\hline 2 & & V1B & 35 & HP 2010 & $\mathrm{~N} 30^{\circ} 49.698^{\prime}$ & $\mathrm{E} 74^{\circ} 45.515^{\prime}$ & 7.7 & 768 & 0.35 & 294 & 216 & 28 & 66 & 0.010 & 0.020 \\
\hline 3 & & V1C & 40 & HP 2010 & $\mathrm{~N} 30^{\circ} 49.711^{\prime}$ & $\mathrm{E} 74^{\circ} 45.519^{\prime}$ & 7.3 & 472 & 0.25 & 236 & 158 & 24 & 46 & 0.010 & 0.010 \\
\hline 4 & & V1D & 45 & HP 2013 & $\mathrm{~N} 30^{\circ} 49.720^{\prime}$ & E74 $45.509^{\prime}$ & 7.9 & 892 & 0.55 & 426 & 254 & 30 & 92 & 0.010 & 0.010 \\
\hline 5 & & V1E & 42 & HP 2008 & $\mathrm{~N} 30^{\circ} 49.726^{\prime}$ & $\mathrm{E} 74^{\circ} 45.512^{\prime}$ & 7.7 & 746 & 0.45 & 314 & 238 & 32 & 86 & 0.011 & 0.010 \\
\hline 6 & & V1F & 40 & HP 2010 & $\mathrm{~N} 30^{\circ} 49.736^{\prime}$ & E74 $45.489^{\prime}$ & 8.0 & 1090 & 1.20 & 458 & 184 & 26 & 130 & 0.010 & 0.010 \\
\hline 7 & & V1G & 40 & HP 2010 & $\mathrm{~N} 30^{\circ} 49.716^{\prime}$ & E74 $45.476^{\prime}$ & 8.1 & 1160 & 1.00 & 514 & 278 & 42 & 156 & 0.010 & 0.010 \\
\hline 8 & & $\mathrm{~V} 1 \mathrm{H}$ & 30 & HP 2009 & $\mathrm{~N} 30^{\circ} 49.724^{\prime}$ & $\mathrm{E} 74^{\circ} 45.460^{\prime}$ & 8.0 & 1120 & 0.85 & 470 & 166 & 28 & 114 & 0.010 & 0.020 \\
\hline 9 & & V1I & 85 & Motor 2013 & $\mathrm{~N} 30^{\circ} 49.711^{\prime}$ & $\mathrm{E} 74^{\circ} 45.457^{\prime}$ & 7.9 & 812 & 0.50 & 398 & 234 & 40 & 98 & 0.010 & 0.010 \\
\hline 10 & & V1J & 25 & HP 1997 & $\mathrm{~N} 30^{\circ} 49.713^{\prime}$ & $\mathrm{E} 74^{\circ} 45.480^{\prime}$ & 7.7 & 794 & 0.35 & 360 & 212 & 34 & 82 & 0.020 & 0.010 \\
\hline 11 & & V1K & 550 & Motor 2016 & $\mathrm{~N} 30^{\circ} 49.745^{\prime}$ & $\mathrm{E} 74^{\circ} 45.477^{\prime}$ & 7.5 & 658 & 0.25 & 282 & 148 & 26 & 74 & 0.012 & 0.010 \\
\hline 12 & & V1L & 225 & Motor 2002 & $\mathrm{~N} 30^{\circ} 49.762^{\prime}$ & $\mathrm{E} 74^{\circ} 45.464^{\prime}$ & 8.0 & 1100 & 1.10 & 426 & 426 & 38 & 146 & 0.010 & 0.010 \\
\hline 13 & & V1M & 125 & HP 2002 & $\mathrm{~N} 30^{\circ} 49.665^{\prime}$ & $\mathrm{E} 74^{\circ} 45.491^{\prime}$ & 7.6 & 642 & 0.45 & 318 & 270 & 34 & 82 & 0.010 & 0.020 \\
\hline 14 & & V1N & 110 & HP 2012 & $\mathrm{~N} 30^{\circ} 49.685^{\prime}$ & $\mathrm{E} 74^{\circ} 45.490^{\prime}$ & 7.9 & 826 & 0.40 & 390 & 142 & 20 & 78 & 0.010 & 0.010 \\
\hline 15 & & V1O & 100 & HP 2012 & $\mathrm{~N} 30^{\circ} 49.730^{\prime}$ & $\mathrm{E} 74^{\circ} 45.515^{\prime}$ & 7.6 & 784 & 0.35 & 330 & 296 & 38 & 74 & 0.010 & 0.010 \\
\hline 16 & V2 & V2A & 120 & Motor 2012 & $\mathrm{~N} 30^{\circ} 55.186^{\prime}$ & $\mathrm{E} 74^{\circ} 35.320^{\prime}$ & 7.5 & 336 & 0.25 & 214 & 248 & 32 & 56 & 0.010 & 0.010 \\
\hline 17 & & V2B & 160 & Motor 2006 & $\mathrm{~N} 30^{\circ} 55.67^{\prime}$ & $\mathrm{E} 74^{\circ} 35.120^{\prime}$ & 7.1 & 324 & 0.35 & 178 & 192 & 26 & 40 & 0.010 & 0.010 \\
\hline 18 & & $\mathrm{~V} 2 \mathrm{C}$ & 120 & Motor 2002 & $\mathrm{~N} 30^{\circ} 55.837^{\prime}$ & $\mathrm{E} 74^{\circ} 35.855^{\prime}$ & 7.5 & 340 & 0.25 & 228 & 216 & 30 & 64 & 0.010 & 0.010 \\
\hline 19 & & V2D & 180 & Motor 2002 & $\mathrm{~N} 30^{\circ} 55.001^{\prime}$ & $\mathrm{E} 74^{\circ} 35.931^{\prime}$ & 7.4 & 376 & 0.40 & 234 & 250 & 36 & 78 & 0.010 & 0.010 \\
\hline 20 & & V2E & 140 & Motor 2015 & $\mathrm{~N} 30^{\circ} 55.296^{\prime}$ & $\mathrm{E} 74^{\circ} 35.870^{\prime}$ & 7.7 & 682 & 0.55 & 326 & 494 & 52 & 88 & 0.012 & 0.010 \\
\hline 21 & & $\mathrm{~V} 2 \mathrm{~F}$ & 220 & Motor 2011 & $\mathrm{~N} 30^{\circ} 55.590^{\prime}$ & $\mathrm{E} 74^{\circ} 35.156^{\prime}$ & 7.6 & 354 & 0.45 & 260 & 238 & 30 & 72 & 0.010 & 0.010 \\
\hline 22 & & V2G & 150 & Motor 2010 & $\mathrm{~N} 30^{\circ} 55.208^{\prime}$ & $\mathrm{E} 74^{\circ} 35.081^{\prime}$ & 8.1 & 1380 & 0.65 & 526 & 850 & 118 & 142 & 0.011 & 0.010 \\
\hline 23 & & $\mathrm{~V} 2 \mathrm{H}$ & 60 & Motor 1997 & $\mathrm{~N} 30^{\circ} 55.025^{\prime}$ & $\mathrm{E} 74^{\circ} 35.767^{\prime}$ & 8.0 & 1190 & 0.50 & 468 & 730 & 94 & 126 & 0.010 & 0.010 \\
\hline 24 & V3 & V3A & 220 & Motor 2004 & $\mathrm{~N} 30^{\circ} 49.674^{\prime}$ & $\mathrm{E} 74^{\circ} 23.432^{\prime}$ & 7.8 & 634 & 0.45 & 352 & 526 & 72 & 98 & 0.010 & 0.010 \\
\hline 25 & & V3B & 230 & Motor 2011 & $\mathrm{~N} 30^{\circ} 49.772^{\prime}$ & $\mathrm{E} 74^{\circ} 23.198^{\prime}$ & 7.2 & 286 & 0.25 & 178 & 194 & 26 & 38 & 0.010 & 0.010 \\
\hline 26 & & $\mathrm{~V} 3 \mathrm{C}$ & 100 & HP 2000 & $\mathrm{~N} 30^{\circ} 49.236^{\prime}$ & $\mathrm{E} 74^{\circ} 23.638^{\prime}$ & 7.9 & 652 & 0.40 & & 470 & 54 & 82 & 0.010 & 0.010 \\
\hline 27 & & V3D & 180 & Motor 2011 & $\mathrm{~N} 30^{\circ} 49.724^{\prime}$ & $\mathrm{E} 74^{\circ} 23.592^{\prime}$ & 7.7 & 53 & & 288 & 446 & 50 & 78 & 0.010 & 0.010 \\
\hline 28 & & V3E & 70 & HP 2015 & $\mathrm{~N} 30^{\circ} 4$ & $\mathrm{E} 74^{\circ} 23.776^{\prime}$ & 7.8 & 56 & & & & 2 & 96 & & 0.010 \\
\hline 29 & & V3F & 225 & Mot & $\mathrm{N} 30^{\circ} 49.925^{\prime}$ & $\mathrm{E} 74^{\circ} 23.078^{\prime}$ & 7.5 & 31 & & & 252 & 50 & 68 & & 0.010 \\
\hline 30 & & V3G & 220 & Motc & N30 & E74 & 7.0 & 246 & & 162 & 178 & 24 & 32 & 0.010 & 0.010 \\
\hline 31 & V4 & V4A & 200 & Motor 2007 & 1.804' & $623^{\prime}$ & 7.9 & 1380 & 0.65 & 322 & 264 & 36 & 178 & 0.010 & 0.010 \\
\hline 32 & & V4B & 350 & Motor 2010 & $\mathrm{~N} 30^{\circ} 51.848^{\prime}$ & $5.612^{\prime}$ & 8.2 & 1740 & 0.85 & 458 & 312 & 44 & 260 & 0.010 & 0.010 \\
\hline 33 & & $\mathrm{~V} 4 \mathrm{C}$ & 350 & Motor 2009 & $\mathrm{~N} 30^{\circ} 51.870^{\prime}$ & $\mathrm{E}^{\prime} 4^{\circ} 55.595^{\prime}$ & 7.9 & 1400 & 0.70 & 316 & 248 & 30 & 186 & 0.010 & 0.010 \\
\hline 34 & & V4D & 310 & Motor 2016 & $1.865^{\prime}$ & $\mathrm{E}^{\circ} 4^{\circ} 55.609^{\prime}$ & 8.1 & 1610 & 1.00 & 432 & 276 & 38 & 234 & 0.020 & 0.010 \\
\hline 35 & & V4E & 250 & Motor 2013 & $\mathrm{~N} 30^{\circ} 51.861^{\prime}$ & E74 $55.584^{\prime}$ & 7.8 & 1490 & 0.80 & 368 & 250 & 34 & 192 & 0.010 & 0.010 \\
\hline 36 & & V4F & 200 & Motor 2007 & $\mathrm{~N} 30^{\circ} 51.839^{\prime}$ & $\mathrm{E} 74^{\circ} 55.626^{\prime}$ & 8.0 & 1550 & 1.00 & 394 & 288 & 40 & 216 & 0.010 & 0.010 \\
\hline 37 & & V4G & 300 & Motor 2013 & $\mathrm{~N} 30^{\circ} 51.922^{\prime}$ & E74 ${ }^{\circ}$ & 7.9 & 1420 & 0.75 & 336 & 254 & 32 & 198 & 0.020 & 0.010 \\
\hline 38 & & V4H & 700 & Motor 2009 & $\mathrm{~N} 30^{\circ} 51.971^{\prime}$ & $\mathrm{E} 74^{\circ} 55.591^{\prime}$ & 7.6 & 534 & 0.30 & 218 & 62 & 16 & 54 & 0.010 & 0.010 \\
\hline 39 & & V4I & 330 & Motor 2014 & $\mathrm{~N} 30^{\circ} 52.010^{\prime}$ & $\mathrm{E} 74^{\circ} 55.586^{\prime}$ & 7.8 & 996 & 0.45 & 280 & 142 & 20 & 86 & 0.010 & 0.010 \\
\hline 40 & & $\mathrm{~V} 4 \mathrm{~J}$ & 240 & Motor 2015 & $\mathrm{~N} 30^{\circ} 51.988^{\prime}$ & $\mathrm{E} 74^{\circ} 55.608^{\prime}$ & 8.0 & 1390 & 0.75 & 346 & 170 & 24 & 168 & 0.011 & 0.010 \\
\hline 41 & & V4K & 250 & Motor 2014 & $\mathrm{~N} 30^{\circ} 51.009^{\prime}$ & $\mathrm{E} 74^{\circ} 55.638^{\prime}$ & 7.9 & 1170 & 0.55 & 221 & 164 & 26 & 132 & 0.010 & 0.016 \\
\hline 42 & & V4L & 350 & Motor 2016 & $\mathrm{~N} 30^{\circ} 51.036^{\prime}$ & $\mathrm{E} 74^{\circ} 55.637^{\prime}$ & 7.8 & 1120 & 0.65 & 274 & 138 & 22 & 116 & 0.010 & 0.010 \\
\hline 43 & & V4M & 750 & Motor 2015 & $\mathrm{~N} 30^{\circ} 52.054^{\prime}$ & $\mathrm{E} 74^{\circ} 55.638^{\prime}$ & 7.5 & 652 & 0.35 & 230 & 84 & 16 & 72 & 0.012 & 0.010 \\
\hline 44 & & V4N & 300 & Motor 2008 & $\mathrm{~N} 30^{\circ} 52.033^{\prime}$ & $\mathrm{E} 74^{\circ} 55.616^{\prime}$ & 7.9 & 1080 & 0.50 & 270 & 132 & 20 & 84 & 0.010 & 0.010 \\
\hline 45 & & $\mathrm{~V} 4 \mathrm{O}$ & 300 & Motor 2006 & $\mathrm{~N} 30^{\circ} 52.019^{\prime}$ & $\mathrm{E} 74^{\circ} 55.623^{\prime}$ & 7.8 & 1040 & 0.45 & 256 & 118 & 20 & 78 & 0.010 & 0.010 \\
\hline 46 & V5 & V5A & 200 & Motor 2006 & $\mathrm{~N} 30^{\circ} 56.907^{\prime}$ & $\mathrm{E} 74^{\circ} 37.609^{\prime}$ & 8.1 & 1190 & 0.65 & 348 & 712 & 126 & 164 & 0.010 & 0.010 \\
\hline 47 & & V5B & 250 & Motor 2007 & $\mathrm{~N} 30^{\circ} 57.003^{\prime}$ & $\mathrm{E} 74^{\circ} 37.624^{\prime}$ & 7.5 & 492 & 0.25 & 220 & 276 & 34 & 46 & 0.010 & 0.010 \\
\hline 48 & & V5C & 200 & Motor 2010 & $\mathrm{~N} 30^{\circ} 56.878^{\prime}$ & $\mathrm{E} 74^{\circ} 37.578^{\prime}$ & 7.7 & 532 & 0.30 & 246 & 218 & 30 & 58 & 0.010 & 0.010 \\
\hline 49 & V6 & V6A & 60 & HP 2009 & $\mathrm{~N} 30^{\circ} 55.001^{\prime}$ & $\mathrm{E} 74^{\circ} 31.193^{\prime}$ & 8.1 & 1300 & 1.10 & 542 & 716 & 98 & 324 & 0.010 & 0.010 \\
\hline 50 & & V6B & 500 & Motor 2017 & $\mathrm{~N} 30^{\circ} 55.001^{\prime}$ & $\mathrm{E} 74^{\circ} 31.193^{\prime}$ & 7.0 & 284 & 0.25 & 150 & 66 & 14 & 38 & 0.010 & 0.010 \\
\hline 51 & & V6C & 60 & HP 2017 & $\mathrm{~N} 30^{\circ} 55.342^{\prime}$ & $\mathrm{E} 74^{\circ} 31.131^{\prime}$ & 7.4 & 372 & 0.30 & 216 & 258 & 32 & 46 & 0.020 & 0.010 \\
\hline 52 & & V6D & 60 & HP 2017 & $\mathrm{~N} 30^{\circ} 55.327^{\prime}$ & $\mathrm{E} 74^{\circ} 31.082^{\prime}$ & 7.5 & 486 & 0.40 & 258 & 294 & 36 & 62 & 0.010 & 0.010 \\
\hline 53 & & V6E & 65 & HP 2010 & $\mathrm{~N} 30^{\circ} 55.141^{\prime}$ & $\mathrm{E} 74^{\circ} 31.187^{\prime}$ & 7.5 & 492 & 0.35 & 284 & 360 & 42 & 86 & 0.010 & 0.016 \\
\hline 54 & & V6F & 50 & HP 2016 & $\mathrm{~N} 30^{\circ} 55.213^{\prime}$ & $\mathrm{E} 74^{\circ} 31.464^{\prime}$ & 7.8 & 910 & 0.45 & 362 & 488 & 54 & 112 & 0.010 & 0.010 \\
\hline 55 & & V6G & 80 & Motor 2002 & $\mathrm{~N} 30^{\circ} 55.202^{\prime}$ & $\mathrm{E} 74^{\circ} 31.462^{\prime}$ & 7.9 & 874 & 0.60 & 330 & 536 & 72 & 98 & 0.010 & 0.010 \\
\hline 56 & & V6H & 40 & HP 2001 & $\mathrm{~N} 30^{\circ} 55.049^{\prime}$ & $\mathrm{E} 74^{\circ} 31.193^{\prime}$ & 7.7 & 622 & 0.45 & 318 & 470 & 52 & 84 & 0.012 & 0.010 \\
\hline 57 & & V6I & 80 & Motor 2005 & $\mathrm{~N} 30^{\circ} 55.049^{\prime}$ & $\mathrm{E} 74^{\circ} 31.193^{\prime}$ & 7.4 & 330 & 0.25 & 216 & 242 & 30 & 48 & 0.010 & 0.010 \\
\hline 58 & & V6J & 80 & Motor 2007 & $\mathrm{~N} 30^{\circ} 55.052^{\prime}$ & $\mathrm{E} 74^{\circ} 31.197^{\prime}$ & 7.6 & 548 & 0.40 & 290 & 432 & 48 & 76 & 0.010 & 0.010 \\
\hline 59 & & V6K & 60 & Motor 2013 & $\mathrm{~N} 30^{\circ} 55.063^{\prime}$ & $\mathrm{E} 74^{\circ} 31.207^{\prime}$ & 7.9 & 616 & 0.50 & 374 & 450 & 56 & 92 & 0.010 & 0.010 \\
\hline 60 & & V6L & 220 & Motor 2012 & $\mathrm{~N} 30^{\circ} 55.060^{\prime}$ & $\mathrm{E} 74^{\circ} 31.203^{\prime}$ & 7.3 & 320 & 0.30 & 166 & 178 & 26 & 40 & 0.010 & 0.010 \\
\hline 61 & & V6M & 200 & Motor 2015 & $\mathrm{~N} 30^{\circ} 55.038^{\prime}$ & $\mathrm{E} 74^{\circ} 31.203^{\prime}$ & 7.5 & 346 & 0.35 & 178 & 190 & 28 & 44 & 0.011 & 0.010 \\
\hline 62 & & V6N & 160 & Motor 2012 & $\mathrm{~N} 30^{\circ} 55.027^{\prime}$ & $\mathrm{E} 74^{\circ} 31.189^{\prime}$ & 7.4 & 358 & 0.25 & 192 & 236 & 32 & 68 & 0.010 & 0.010 \\
\hline 63 & & $\mathrm{~V} 6 \mathrm{O}$ & 255 & Motor 2017 & $\mathrm{~N} 30^{\circ} 55.018^{\prime}$ & $\mathrm{E} 74^{\circ} 31.208^{\prime}$ & 7.1 & 312 & 0.30 & 170 & 184 & 26 & 50 & 0.010 & 0.010 \\
\hline 64 & & V6P & 65 & Motor 2007 & $\mathrm{~N} 30^{\circ} 54.707^{\prime}$ & $\mathrm{E} 74^{\circ} 32.167^{\prime}$ & 7.9 & 720 & 0.65 & 438 & 546 & 70 & 118 & 0.010 & 0.016 \\
\hline 65 & & V6Q & 80 & Motor 2000 & $\mathrm{~N} 30^{\circ} 54.527^{\prime}$ & $\mathrm{E} 74^{\circ} 32.827^{\prime}$ & 7.7 & 600 & 0.45 & 324 & 458 & 56 & 80 & 0.010 & 0.010 \\
\hline 66 & & V6R & 70 & HP 2001 & $\mathrm{~N} 30^{\circ} 54.948^{\prime}$ & $\mathrm{E} 74^{\circ} 32.203^{\prime}$ & 8.1 & 1090 & 0.85 & 456 & & 90 & 134 & 0.010 & 0.010 \\
\hline
\end{tabular}




\section{RESULTS AND DISCUSSION}

Impurities in drinking water were detected by using improved analytical methods to ascertain the quality of drinking water. Table-1 denotes the results of physico-chemical parameters and heavy metals present in drinking water samples collected from selected sampling sites across district Ferozepur of Punjab state, India.

The results of some physico-chemical parameters like $\mathrm{pH}$, TDS, alkalinity, total hardness, fluoride, chloride and some heavy metals like arsenic and lead are shown in Table-1. As per WHO guidelines, $\mathrm{pH}$ of drinking water should lie in between 6.5-8.5. The $\mathrm{pH}$ of water samples was found to lie in between 7.0 to 8.2 with mean $\mathrm{pH} 7.71$ which was slightly alkaline. The minimum and maximum $\mathrm{pH}$ value of 7.0 and 8.2 was observed in the sample ID V3G and ID V4B, respectively.

High TDS values were observed at many locations. The acceptable limit of TDS in water is $1000 \mathrm{mg} / \mathrm{L}$ as per WHO standards. Twenty one samples (32\%) were found to have TDS more than $1000 \mathrm{mg} / \mathrm{L}$ with maximum TDS of $1740 \mathrm{mg} / \mathrm{L}$ in sample no. 32 (sub-tehsil Talwandi Bhai). The broad agricultural practices and large extraction of groundwater leads to high concentrations of dissolved materials which further increase value of TDS. High TDS is due to $\mathrm{Na}^{+}, \mathrm{K}^{+}, \mathrm{Ca}^{2+}, \mathrm{Mg}^{2+}$, $\mathrm{CO}_{3}{ }^{2-}, \mathrm{Cl}^{-}$, etc. ions present in water.

The maximum permissible limit of total hardness in water is $600 \mathrm{mg} / \mathrm{L}$ as per WHO guidelines. About $88 \%$ of samples were found to have total hardness less than the maximum permissible limit. The total hardness of sample ID V2G was more than the permissible limit with maximum value of 850 $\mathrm{mg} / \mathrm{L}$.

The permissible limit for calcium is $75 \mathrm{mg} / \mathrm{L}$ as per $\mathrm{WHO}$ guidelines. Presence of calcium in sample ID V5A was more than the permissible limit with maximum value of $126 \mathrm{mg} / \mathrm{L}$. As per WHO guidelines, the permissible limit of arsenic content is $0.010 \mathrm{mg} / \mathrm{L}$ and there are $19 \%$ samples found to have arsenic content more than permissible limit. Around $0.09 \%$ samples found to have lead content beyond permissible limit that is $0.010 \mathrm{mg} / \mathrm{L}$. Table- 2 denotes the summary of water quality parameters of studied area.

\section{Statistical analysis}

Fluoride analysis: Groundwater samples contained fluoride concentration ranges from 0.25 to $1.20 \mathrm{mg} / \mathrm{L}$ with mean value and SD of $0.52 \mathrm{mg} / \mathrm{L}$ and 0.08575 , respectively. As per analysis results, it was noted that no sample exceeded the acceptable limit (Table-3). The minimum fluoride concentration $(0.6 \mathrm{mg} / \mathrm{L})$ which prevents dental caries and encourages the development of bones and is unfit for drinking purpose. About $69.9 \%$ of total samples found to have fluoride concentration below minimum fluoride concentration required. Talwandi Bhai is the location where most of the samples having fluoride concentration within permissible limits. Table-3 and Fig. 2 denotes the frequency distribution of fluoride against sampling area.

The study also revealed that the fluoride dissolution increases with respect to depth as there could be an increase in the geo-

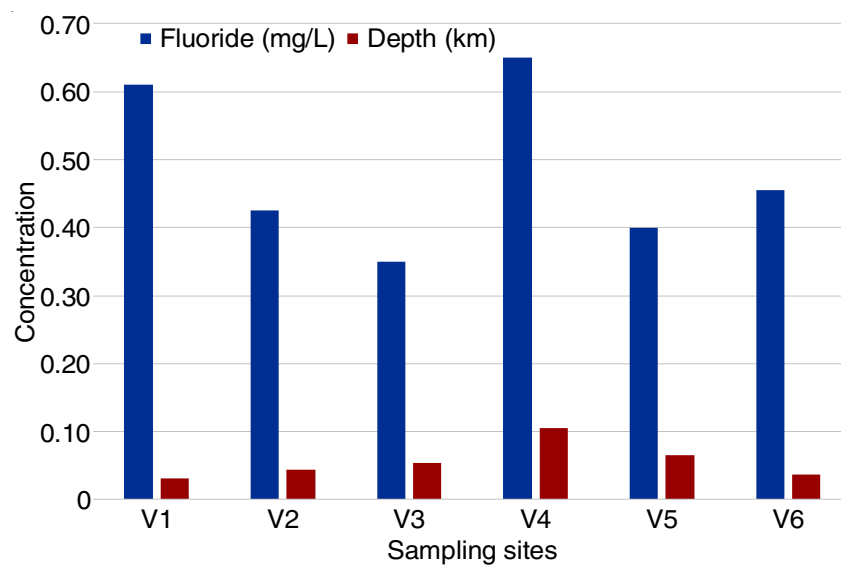

Fig. 2. Concentration of fluoride and depth at various sampling sites

TABLE-2

SUMMARY OF WATER QUALITY PARAMETERS OF STUDIED AREA

\begin{tabular}{ccccccccccc}
\hline $\begin{array}{c}\text { Name of } \\
\text { village }\end{array}$ & $\begin{array}{c}\text { No. of } \\
\text { sample }\end{array}$ & $\begin{array}{c}\text { TDS } \\
(\mathrm{mg} / \mathrm{L})\end{array}$ & $\mathrm{F}(\mathrm{mg} / \mathrm{L})$ & $\mathrm{pH}$ & $\begin{array}{c}\text { Alkalinity } \\
(\mathrm{mg} / \mathrm{L})\end{array}$ & $\begin{array}{c}\text { Total hardness } \\
(\mathrm{mg} / \mathrm{L})\end{array}$ & $\begin{array}{c}\mathrm{Ca}^{2+} \\
(\mathrm{mg} / \mathrm{L})\end{array}$ & $\begin{array}{c}\mathrm{Cl}^{-} \\
(\mathrm{mg} / \mathrm{L})\end{array}$ & $\begin{array}{c}\mathrm{As}^{3+} \\
(\mathrm{mg} / \mathrm{L})\end{array}$ & $\begin{array}{c}\mathrm{Pb}^{2+} \\
(\mathrm{mg} / \mathrm{L})\end{array}$ \\
\hline V1 & 15 & $472-1160$ & $0.25-1.20$ & $7.3-8.1$ & $236-514$ & $142-426$ & $20-42$ & $46-156$ & $0.010-0.020$ & $0.010-0.010$ \\
V2 & 8 & $324-1380$ & $0.25-0.65$ & $7.1-8.1$ & $178-526$ & $192-850$ & $26-118$ & $40-142$ & $0.010-0.012$ & $0.010-0.010$ \\
V3 & 7 & $246-652$ & $0.25-0.45$ & $7.0-7.9$ & $162-352$ & $178-526$ & $24-72$ & $32-98$ & $0.010-0.011$ & $0.010-0.010$ \\
V4 & 15 & $534-1740$ & $0.30-1.00$ & $7.5-8.2$ & $221-458$ & $62-312$ & $16-44$ & $54-260$ & $0.010-0.020$ & $0.010-0.010$ \\
V5 & 3 & $492-1190$ & $0.25-0.65$ & $7.5-8.1$ & $220-348$ & $218-712$ & $30-126$ & $58-164$ & $0.010-0.010$ & $0.010-0.010$ \\
V6 & 18 & $312-1300$ & $0.25-1.10$ & $7.0-8.1$ & $150-542$ & $66-716$ & $14-98$ & $38-324$ & $0.010-0.020$ & $0.010-0.016$ \\
Total & 66 & $246-1740$ & $0.25-1.20$ & $7.0-8.2$ & $150-542$ & $62-850$ & $14-126$ & $32-324$ & $0.010-0.020$ & $0.010-0.010$ \\
Mean & & 793.82 & 0.52 & 7.71 & 314.20 & 305.06 & 40.64 & 101.82 & 0.0108 & 0.0107 \\
\hline
\end{tabular}

TABLE-3

FREQUENCY DISTRIBUTION OF WATER QUALITY PARAMETERS OF STUDIED AREA

\begin{tabular}{|c|c|c|c|c|c|c|c|c|c|c|c|c|c|c|}
\hline \multirow[b]{2}{*}{$\begin{array}{c}\text { S. } \\
\text { No. }\end{array}$} & \multirow[b]{2}{*}{$\begin{array}{c}\text { Name } \\
\text { of } \\
\text { village }\end{array}$} & \multirow[b]{2}{*}{$\begin{array}{l}\text { No. of } \\
\text { sample }\end{array}$} & \multicolumn{3}{|c|}{$\mathrm{F}(\mathrm{mg} / \mathrm{L})$} & \multicolumn{3}{|c|}{$\mathrm{Cl}^{-}(\mathrm{mg} / \mathrm{L})$} & \multicolumn{3}{|c|}{$\mathrm{As}^{3+}(\mathrm{mg} / \mathrm{L})$} & \multicolumn{3}{|c|}{$\mathrm{Pb}^{2+}(\mathrm{mg} / \mathrm{L})$} \\
\hline & & & $<1.0$ & $1.0-1.5$ & $>1.5$ & $<250$ & $\begin{array}{l}250- \\
1000\end{array}$ & $>1000$ & $<0.010$ & $\begin{array}{l}\text { Equal } \\
\text { to } \\
0.010\end{array}$ & $>0.010$ & $<0.010$ & $\begin{array}{l}\text { Equal } \\
\text { to } \\
0.010\end{array}$ & $>0.010$ \\
\hline 1 & V1 & 15 & 11 & 4 & Nil & 15 & Nil & Nil & Nil & 12 & 3 & Nil & 12 & 3 \\
\hline 2 & V2 & 8 & 8 & Nil & Nil & 8 & Nil & Nil & Nil & 6 & 2 & Nil & 8 & Nil \\
\hline 3 & V3 & 7 & 7 & Nil & Nil & 7 & Nil & Nil & Nil & 6 & 1 & Nil & 7 & Nil \\
\hline 4 & V4 & 15 & 13 & 2 & Nil & 15 & Nil & Nil & Nil & 11 & 4 & Nil & 14 & 1 \\
\hline 5 & V5 & 3 & 3 & Nil & Nil & 3 & Nil & Nil & Nil & 3 & Nil & Nil & 3 & Nil \\
\hline 6 & V6 & 18 & 17 & 1 & Nil & 18 & Nil & Nil & Nil & 15 & 3 & Nil & 16 & 2 \\
\hline
\end{tabular}


thermal gradient. Although the observation on the interdependency between depth and fluoride concentration was positively correlated (Fig. 3), the lower fluoride concentration in groundwater may be associated with the mixing of water with different chemical compositions. The enrichment of fluoride is in shallow groundwater sources because of evapotranspiration and thus, the concentration of fluoride at deep groundwater sources is less.

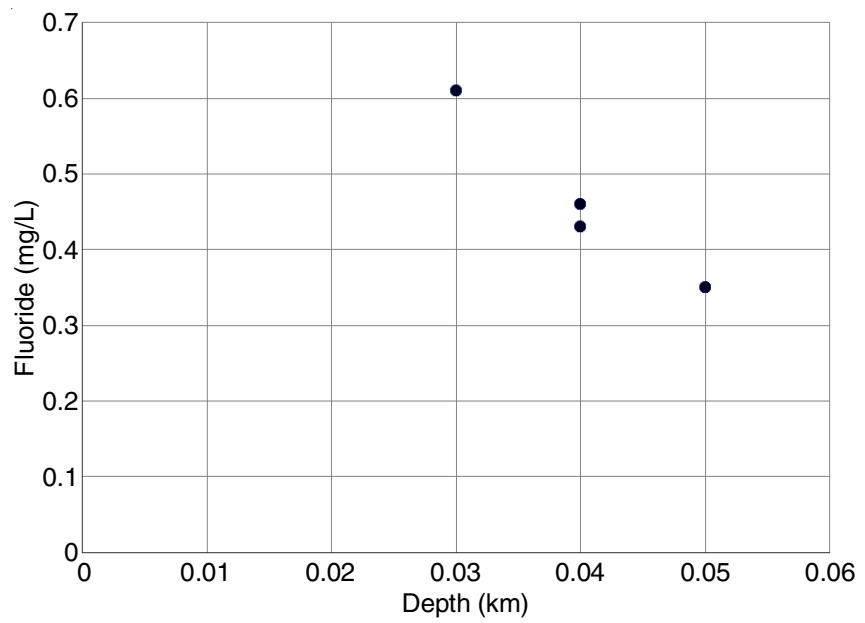

Fig. 3. Relation $\mathrm{b} / \mathrm{w}$ depth and fluoride at various sampling sites

Chloride analysis: Groundwater samples contained 32 to $324 \mathrm{mg} / \mathrm{L}$ of chloride concentration with mean and SD value of $101.82 \mathrm{mg} / \mathrm{L}$ and 26.266 , respectively. The analysis data showed that no sample exceeded the acceptable limit (Table3). $250 \mathrm{mg} / \mathrm{L}$ is the minimum chloride concentration which is needed to stop the harmful effects on metal pipes and agricultural implements and $96.97 \%$ of total samples found to have chloride concentration below minimum concentration.
The study showed that chloride concentration increases with increase in depth (Table-4). The relation between depth and chloride concentration is shown in Fig. 4. Due to weather conditions, the process of leaching of chloride from rocks into soil and water is done. Due to mobility characteristic of chloride ion, it is transported to closed oceans, hence, the concentration of chloride is high at deep groundwater sources.

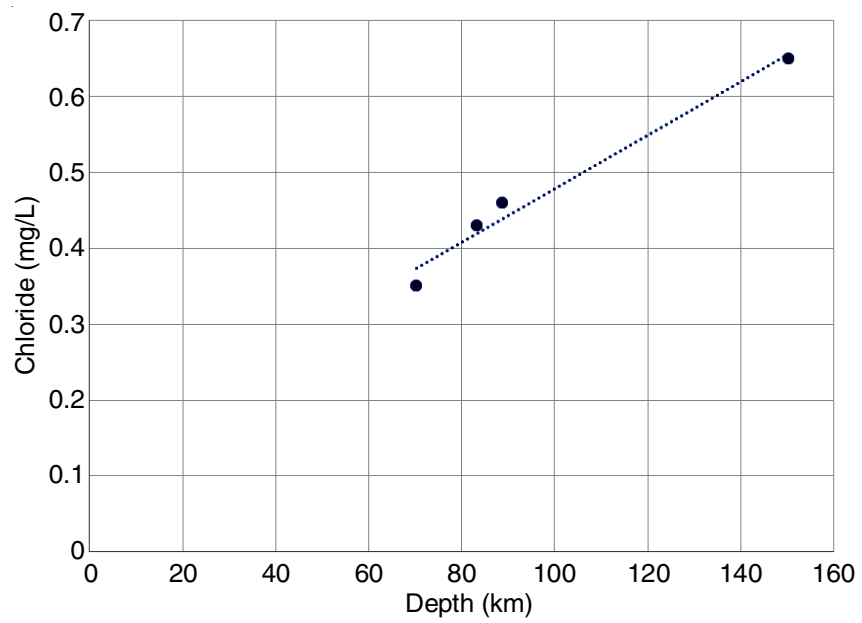

Fig. 4. Relation $\mathrm{b} / \mathrm{w}$ concentration of chloride and depth

Correlation analysis: The fluoride and chloride concentrations in groundwater showed significant relationship with $\mathrm{pH}$ and depth.

Effect of $\mathbf{p H}$ on fluoride and chloride: Fluoride concentration has showed a positive correlation with $\mathrm{pH}$ whereas chloride showed slightly negative trend with $\mathrm{pH}\left(\mathrm{R}^{2}=0.9975\right.$ and 0.6804 , respectively) (Table-5). The positive correlation between $\mathrm{pH} \&$ fluoride and $\mathrm{pH} \&$ chloride levels might be

\begin{tabular}{|c|c|c|c|c|c|c|c|c|c|}
\hline \multicolumn{10}{|c|}{$\begin{array}{c}\text { TABLE-4 } \\
\text { STATISTICAL ANALYSIS OF WATER SAMPLES }\end{array}$} \\
\hline $\begin{array}{l}\text { Name of } \\
\text { village }\end{array}$ & $\begin{array}{l}\text { No. of } \\
\text { samples }\end{array}$ & $\mathrm{F}(\mathrm{mg} / \mathrm{L})$ & Range & Mean & SD & $\mathrm{Cl}^{-}(\mathrm{mg} / \mathrm{L})$ & Range & Mean & SD \\
\hline V1 & 15 & $0.25-1.20$ & 0.95 & 0.61 & 0.33922 & $46-156$ & 110 & 96.0000 & 30.6874 \\
\hline V2 & 8 & $0.25-0.65$ & 0.40 & 0.425 & 0.14142 & $40-142$ & 102 & 83.2500 & 34.7224 \\
\hline V3 & 7 & $0.25-0.45$ & 0.20 & 0.35 & 0.08660 & $32-98$ & 66 & 70.2857 & 26.266 \\
\hline V4 & 15 & $0.30-1.00$ & 0.70 & 0.65 & 0.21630 & $54-260$ & 206 & 150.267 & 65.6826 \\
\hline V5 & 3 & $0.25-0.65$ & 0.40 & 0.4 & 0.21794 & $58-164$ & 106 & 89.3333 & 64.9410 \\
\hline V6 & 18 & $0.25-1.10$ & 0.85 & 0.456 & 0.22485 & $38-324$ & 286 & 88.8889 & 65.1883 \\
\hline $\begin{array}{l}\text { Name of } \\
\text { village }\end{array}$ & $\begin{array}{c}\text { No. of } \\
\text { samples }\end{array}$ & $\mathrm{As}^{3+}(\mathrm{mg} / \mathrm{L})$ & Range & Mean & SD & $\mathrm{Pb}^{2+}(\mathrm{mg} / \mathrm{L})$ & Range & Mean & SD \\
\hline V1 & 15 & $0.010-0.020$ & 0.010 & 0.010 & 0.002499 & $0.010-0.010$ & 0.000 & 0.012 & 0.00414 \\
\hline V2 & 8 & $0.010-0.012$ & 0.002 & 0.010 & 0.000744 & $0.010-0.010$ & 0.000 & 0.010 & 0.00000 \\
\hline V3 & 7 & $0.010-0.011$ & 0.001 & 0.010 & 0.000377 & $0.010-0.010$ & 0.000 & 0.010 & 0.00000 \\
\hline V4 & 15 & $0.010-0.020$ & 0.010 & 0.011 & 0.003481 & $0.010-0.010$ & 0.000 & 0.010 & 0.00154 \\
\hline V5 & 3 & $0.010-0.010$ & 0.000 & 0.010 & 0.000000 & $0.010-0.010$ & 0.000 & 0.010 & 0.00000 \\
\hline V6 & 18 & $0.010-0.020$ & 0.010 & 0.010 & 0.002371 & $0.010-0.016$ & 0.006 & 0.010 & 0.00194 \\
\hline
\end{tabular}

\section{TABLE-5}

ANOVA: SHOWING EFFECT OF pH ON FLUORIDE \& CHLORIDE IN GROUNDWATER

\begin{tabular}{lccccccc}
\hline & & DF & Sum of squares & Adjusted $R^{2}$ & Mean square & F-value & $F_{\text {critical }}$ \\
\hline Fluoride & Model & 1 & 156.2206 & 0.997561 & 156.2206 & 10309.96 \\
Fluoride & Error & 10 & 0.151524 & & 0.0151524 & \\
Fluoride & Total & 11 & 156.372124 & & & \\
Chloride & Model & 1 & 23570.8296 & 0.6804 & 23570.8296 & 61.01527 \\
Chloride & Error & 10 & 3863.1029 & & 386.31029 & \\
Chloride & Total & 11 & 27433.9325 & & & \\
\hline
\end{tabular}


due to the release of hydroxyl and bicarbonate ions simultaneously during the leaching and dissolution process of fluoridebearing minerals in the groundwater. The solubility of fluoride is lowest in the $\mathrm{pH}$ range of 5.0 to 5.6 due to its absorption on the surface of minerals present in clay in sub-surface rocks.

Effect of depth on fluoride and chloride: Fluoride and chloride have showed a negative and positive correlation with depth having $\mathrm{R}^{2}=0.715517$ and 0.720376 , respectively) (Table$6)$. High fluoride and low chloride concentrations have been found in the shallow surface waters, whereas fluoride concentration reduces with increase in depth, on the other hand, chloride concentration increases with increase in depth. Fluoride occurrence is due to geogenic as well as anthropogenic reasons but chloride occurrence is because of its build up during evaporation process in the soil because chloride is not taken up by plants and non-volatile. Chloride moves conservatively in liquid water throughout the hydrologic cycle.

Effect of depth on arsenic and lead: Arsenic and lead showed a negative correlation with depth having $\mathrm{R}^{2}=0.04036$ and 0.215407 , respectively) (Table-7). Arsenic concentration remains constant in majority of the samples and was found within permissible limit in $81 \%$ samples as prescribed by WHO i.e. $0.010 \mathrm{mg} / \mathrm{L}$, whereas the concentration of lead reduces with increase in depth and was found within permissible limit as prescribed by WHO i.e. in this case is $0.010 \mathrm{mg} / \mathrm{L}$.

Water quality index: The quality of groundwater at certain places in Ferozepur district can also be ascertained by calcul- ating water quality index. The methodology for computation of water quality index (WQI) includes following steps:

$$
\mathrm{WQI}=\frac{\Sigma \mathrm{W}_{\mathrm{n}} \mathrm{Q}_{\mathrm{n}}}{\Sigma \mathrm{W}_{\mathrm{n}}}
$$

The quality rating index $\left(\mathrm{Q}_{\mathrm{n}}\right)$ is calculated by using this expression:

$$
\mathrm{Q}_{\mathrm{n}}=\left(\frac{\mathrm{V}_{\mathrm{n}}-\mathrm{V}_{\mathrm{i}}}{\mathrm{S}_{\mathrm{n}}-\mathrm{V}_{\mathrm{i}}}\right) \times 100
$$

where, $V_{n}$ is estimated value of the parameters of groundwater in Ferozpur district; $\mathrm{V}_{\mathrm{i}}$ is the ideal value of parameter in pure water; $\mathrm{V}_{\mathrm{i}}=0$ (except $\mathrm{pH}=7.0$ for all other parameters); $\mathrm{S}_{\mathrm{n}}$ is standard permissible value of parameter of groundwater. The unit weight $\left(\mathrm{W}_{\mathrm{i}}\right)$ for each water quality parameter is calculated by using the following formula:

$$
\mathrm{W}_{\mathrm{n}}=\frac{\mathrm{K}}{\mathrm{S}_{\mathrm{n}}}
$$

where, $\mathrm{K}$ is proportionality constant and can also be calculated by using the following equation:

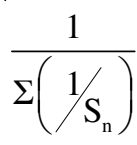

The water quality index of groundwater in Ferozepur district is equal to 107 (Table-8). The value is close to 100 so

TABLE-6

ANOVA: SHOWING EFFECT OF DEPTH ON FLUORIDE \& CHLORIDE IN GROUNDWATER

\begin{tabular}{lccccccc}
\hline & & $\mathrm{DF}$ & Sum of squares & Adjusted $\mathrm{R}^{2}$ & Mean square & F-value & $\mathrm{F}_{\text {critical }}$ \\
\hline Fluoride & Model & 1 & 0.542384 & 0.715517 & 0.542384 & 70.4389 & \\
Fluoride & Error & 10 & 0.077 & & 0.0077 & \\
Fluoride & Total & 11 & 0.619384 & & & 71.99 & \\
Chloride & Model & 1 & 27809.976 & 0.720376 & 27809.976 & 4.9646 \\
Chloride & Error & 10 & 3863.0284 & & 386.3028 & \\
Chloride & Total & 11 & 31673.004 & & & \\
\hline
\end{tabular}

TABLE-7

ANOVA: SHOWING EFFECT OF DEPTH ON ARSENIC \& LEAD IN GROUNDWATER

\begin{tabular}{cccccccc}
\hline & & $\mathrm{DF}$ & Sum of squares & Adjusted $\mathrm{R}^{2}$ & Mean square & F-value & $\mathrm{F}_{\text {critical }}$ \\
\hline Arsenic & Model & 1 & 0.006486 & 0.04036 & 0.006486 & 8.59 & \\
Arsenic & Error & 10 & 0.007554 & & 0.0007554 & \\
Arsenic & Total & 11 & 0.01404 & & & \\
Lead & Model & 1 & 0.00643107 & 0.215407 & 0.006431 & 14.8387 \\
Lead & Error & 10 & 0.00433396 & & 0.000433396 & \\
Lead & Total & 11 & 0.011 & & & \\
\hline
\end{tabular}

TABLE-8

WATER QUALITY INDEX OF GROUND WATER IN FEROZEPUR DISTRICT

\begin{tabular}{lccccccc}
\hline \multicolumn{1}{c}{ Parameters } & $\mathrm{V}_{\mathrm{i}}$ & $\mathrm{S}_{\mathrm{n}}$ & $1 / \mathrm{S}_{\mathrm{n}}$ & $\mathrm{K}$ & $\mathrm{W}_{\mathrm{n}}$ & $\mathrm{Q}_{\mathrm{n}}$ & $\mathrm{W}_{\mathrm{n}} \times \mathrm{Q}_{\mathrm{n}}$ \\
\hline $\mathrm{TDS}(\mathrm{mg} / \mathrm{L})$ & 793.82 & 1000 & 0.001 & 0.00498 & 0.00000498 & 79.3818182 & 0.000395321 \\
$\mathrm{~F}(\mathrm{mg} / \mathrm{L})$ & 0.52 & 1.5 & 0.666666667 & 0.00498 & 0.00332 & 34.66666667 & 0.115093333 \\
$\mathrm{pH}$ & 7.71 & 8.5 & 0.117647059 & 0.00498 & 0.000585882 & 47.172 & 0.027637242 \\
Alkalinity (mg/L) & 314.20 & 120 & 0.008333333 & 0.00498 & 0.0000415 & 261.8308081 & 0.010865979 \\
Total hardness (mg/L) & 305.06 & 600 & 0.001666667 & 0.00498 & 0.0000083 & 50.84343435 & 0.000422001 \\
$\mathrm{Ca}^{2+}(\mathrm{mg} / \mathrm{L})$ & 40.64 & 75 & 0.013333333 & 0.00498 & 0.0000664 & 54.18181333 & 0.003597672 \\
$\mathrm{Cl}^{-}(\mathrm{mg} / \mathrm{L})$ & 101.82 & 250 & 0.004 & 0.00498 & 0.00001992 & 40.727248 & 0.000811287 \\
$\mathrm{As}^{3+}(\mathrm{mg} / \mathrm{L})$ & 0.0108 & 0.010 & 100 & 0.00498 & 0.498 & 108 & 53.784 \\
$\mathrm{~Pb}^{2+}(\mathrm{mg} / \mathrm{L})$ & 0.0107 & 0.010 & 100 & 0.00498 & 0.498 & 107 & 53.286 \\
\hline & & & $\Sigma 1 / \mathrm{S}_{\mathrm{i}}=200.8$ & & $\Sigma \mathrm{W}_{\mathrm{n}}=1.00005$ & & $\Sigma \mathrm{W}_{\mathrm{n}} \times \mathrm{Q}_{\mathrm{n}}=107.229$ \\
\hline
\end{tabular}


the quality of groundwater in Ferozepur district can be categorized under 'Good Quality' water.

\section{Conclusion}

The results of the study revealed that groundwater quality in north-west district of Punjab i.e. Ferozepur is having a small amount of fluoride and chloride contents in groundwater but majority of the samples are within the acceptable limits when the water samples were collected from depth ranging from $100 \mathrm{ft}$ to $360 \mathrm{ft}$. The maximum fluoride concentration has been observed at shallow depths upto $100 \mathrm{ft}$. with decreasing trend afterwards suggesting that fluoride contamination decreases with increase in depth of groundwater beyond $100 \mathrm{ft}$. The studies showed that the chloride serves as a tracer of water movement. Evapotranspiration causes chloride to build up in the soil because chloride is not taken up by plants and is non-volatile. The concentration of chloride was found to be within limits at shallow depths and as well as depth upto $360 \mathrm{ft}$. The value of TDS in $32 \%$ samples was found more than $1000 \mathrm{mg} / \mathrm{L}$ with maximum TDS of $1740 \mathrm{mg} / \mathrm{L}$ in sample at site no. 32 (sub-tehsil Talwandi Bhai). The concentration of arsenic was also found to be within permissible limits of WHO at any depth in $81 \%$ samples while $19 \%$ samples contain arsenic beyond permissible limit, whereas, the concentration of lead decreases with increase in depth. As the depth increases beyond $100 \mathrm{ft}$, concentration of lead lies within permissible limits in most of the samples. ANOVA has been applied on analysis results to study the effect of $\mathrm{pH}$ on fluoride and chloride, depth on fluoride and chloride and depth on arsenic and lead. The results showed that F-value is greater than F-critical in said correlation analysis which is in accor- dance with the results obtained. Also, to adjudge the overall quality of groundwater in Ferozepur district, the water quality index has been calculated on the basis of large number of physico-chemical characteristics of groundwater. This value of water quality index indicates that the groundwater quality in Ferozepur district of Punjab state can be considered of 'Good Quality' when the groundwater is used at the depth ranging from $100 \mathrm{ft}$ to $360 \mathrm{ft}$.

\section{CONFLICT OF INTEREST}

The authors declare that there is no conflict of interests regarding the publication of this article.

\section{REFERENCES}

1. W.J. Cosgrove and D.P. Loucks, Water Resour. Res., 51, 4823 (2015); https://doi.org/10.1002/2014WR016869.

2. H.S. Hundal, R. Kumar, K. Singh and D. Singh, Commun. Soil Sci. Plant Anal., 38, 17 (2007); https://doi.org/10.1080/00103620701588312.

3. S. Singh, N.J. Raju and Ch. Ramakrishna, J. Water Resour. Prot., 7, $572(2015)$; https://doi.org/10.4236/jwarp.2015.77046.

4. N. Khatri and S. Tyagi, Front. Life Sci., 8, 23 (2015); https://doi.org/10.1080/21553769.2014.933716.

5. S. Mitra, Int. J. Ecosystem, 5, 43 (2015); https://doi.org/10.5923/c.ije.201501.06.

6. T. Kanyerere, J. Levy, Y. Xu and J. Saka, Water SA, 38, 581 (2012).

7. D. Sugimoto, Senri Ethnol. Stud., 96, 35 (2017); https://doi.org/10.15021/00008671.

8. N. Rahmanian, S.H.B. Ali, M. Homayoonfard, N.J. Ali, M. Rehan, Y. Sadef and A.S. Nizami, J. Chem., Article ID 716125 (2015).

9. P. Mariappan, V. Yegnaraman and T. Vasudevan, Pollut. Res., 19, 165 (2000). 